\title{
Model of the Mechanical Behavior of Cementitious Matrices Reinforced with Nanomaterials
}

\author{
Victor D. Balopoulos, ${ }^{1}$ Nikolaos Archontas, ${ }^{2}$ and Stavroula J. Pantazopoulou ${ }^{3}$ \\ ${ }^{1}$ Civil Engineering Department, Democritus University of Thrace (DUTh), Xanthi, Greece \\ ${ }^{2}$ Department of Civil \& Environmental Engineering, University of Cyprus, Nicosia, Cyprus \\ ${ }^{3}$ Civil Engineering Department, The Lassonde School of Engineering, York University, Toronto, ON, Canada \\ Correspondence should be addressed to Victor D. Balopoulos; vbalop@civil.duth.gr
}

Received 23 September 2016; Accepted 19 February 2017; Published 20 March 2017

Academic Editor: Peng Zhang

Copyright (C) 2017 Victor D. Balopoulos et al. This is an open access article distributed under the Creative Commons Attribution License, which permits unrestricted use, distribution, and reproduction in any medium, provided the original work is properly cited.

\begin{abstract}
CNTs and CNFs have been introduced as a nanoscale reinforcing material to cementitious composites, for stiffening and strengthening the microstructure. This technology is motivated by the need to control crack initiation in the cementitious gel before it propagates into visible crack formations. Experimental evidence supports this concept; however, testing at the nanoscale may only be conducted through nanoindentation, which has a limited range only providing localized results that cannot be extrapolated to general stress states. To evaluate the restraining action of nanomaterials in the gel microstructure, a computational mechanistic model has been developed where the material phases (gel, nanotubes, and pores) are modeled explicitly allowing for natural randomness in their distribution and orientation. Repeated analysis with identical input data reproduces the statistical scatter observed in laboratory tests on identical material samples. The formulation uses a discrete element approach; the gel structure is represented by a random network of hydrates and successfully reproduces the known trends in mechanical behavior of cementitious materials (pressure and restraint sensitive material behavior) and the small ratio of tensile to compressive strength. Simulations illustrate that it is possible to computationally reproduce the measured properties and behavior of fiber-reinforced cement composites using information from simple laboratory tests.
\end{abstract}

\section{Introduction}

New generations of functionally graded cementitious materials have emerged in recent years designed to meet specific performance objectives. These developments in the materials technology open up the prospects of a new era in construction, where the traditional concrete will be displaced in favor of more durable cementitious materials that possess higher fracture energy and are resilient to tensile deformation. An important milestone towards this objective is the new generation of fiber-reinforced matrices that contain a hybrid mix of fibers as a means to enhance the performance through the synergistic effects imparted by the various types of fibers functioning at the various scales of the FRC microstructure [1]. Hybrid mixes usually contain a cocktail of fibers of different materials (synthetic or metallic) and surface properties, with lengths ranging from a few nanometers (CNTs and CNFs) up to practically any length. In this regard, each fiber length range corresponds to a specific scale of the material: nanofibers can only function to arrest flaws and cracks of size commensurate to their length [2-4]; similarly, microfibers function to restrain the propagation of flaws within the microscale, whereas longer fibers are intended to arrest visible cracks. Naturally, if a crack or flaw surpasses the geometric scale of any given type of fiber, it is expected that the next fiber scale will be mobilized to arrest it. In this stage, smaller fibers are not rendered inactive: each fiber range confines the anchorage of the next, longer scale fiber group. This is macroscopically evident through the enhanced fracture energy of the material; note that the poor tensile resistance of plain cementitious materials is due to the low strain energy storage capacity of the material lattice [5-7]. (The low-strength and limited-range cohesive forces, which bind the various products of cement hydration together in the 
gel, diminish fast with increasing separation distance which occurs under tensile strain; this is manifested by the size effects and the independence of failure strain to transverse confining stress in tension-dominated failures of concrete [5].)

Although the synergistic effects of fibers with reinforcement, as well as synthetic and steel fiber cocktails, have been studied, only recently have pioneering efforts to address the flaws at the nanoscale been made through the addition of nanomaterials to the cementitious mix [2-8]. Note that macroscopic cracking is believed to initiate at the nanoscale in the gel structure. Of the additives that have been considered so far, nanoparticles (e.g., nanosilica) are intended to generate a more compact gel structure where flaws are of smaller size whereas the redundancy generated through compaction effectively causes significant redistribution of stress, thereby partially relieving the stress concentrations that lead to flaw propagation. On the other hand, carbon nanotubes (CNTs) and carbon nanofibers (CNFs), whose operating range is at the nanometer scale, are a form of passive reinforcement of the gel nanostructure, having an analogous role, at that scale, with macroscopic reinforcement in concrete. The mechanistic role of this type of reinforcement has been modeled analytically in [5] and it comprises the mere addition of effective reinforcement contribution to tensile resistance, $\rho_{\mathrm{CNT}} \cdot f_{\mathrm{CNT}}$ in the otherwise plain cementitious material cross section $\left(\rho_{\mathrm{CNT}}\right.$ and $f_{\mathrm{CNT}}$ are the effective area ratio and average stress intensity in the CNT network in the direction of tensile load). Experiments consistently illustrate that these materials effectively mitigate several of the fracturerelated effects enhancing by measurable margin the tensile resistance, fracture energy, and most significantly the stiffness (Young's modulus) of the cementitious matrix $[2,5-8]$.

Studying the nanoreinforced material response to general states of stress requires a calibrated constitutive model that reflects the parametric sensitivities as well as the uncertainties necessarily embedded in the macroscopic properties of the composite. Recall that cementitious materials are amorphous, whereas past works that have published pictures of the nanostructure obtained through electron-scanning microscopy (ESM) reveal that CNTs often are inadequately distributed unless properly treated with surfactants $[3,4]$. At best, the material has a random network of CNTs crossing an amorphous solid structure of hydrates. In this regard, the properties of the material have a statistical rather than a uniquely defined deterministic value. To study the effect of this randomness on the macroscopic mechanical properties and to quantify the strength and stiffness enhancement imparted by the addition of CNTs and CNFs, a discrete modeling framework was developed and further calibrated in this paper, mimicking the function of the gel structure. Thus, a statistically generated, continuous network of flaky dendrites comprising cement hydrates growing from partially hydrated cement grains is the background solid structure of the cementitious material in nanoscale. (Dendrites are the solid phase of the cement gel and interconnect to provide strength and stiffness against load.) Fibers such as CNTs are linear elements that span randomly between nodes of the dendrite mesh. Spaces that are not occupied either by dendrite material or by the nanoreinforcement represent the pores, randomly located throughout the mesh. The idealized solid is subjected to displacement-controlled uniform boundary conditions, thereby simulating various stress states similar to lab tests. In this manner, the model is tested regarding its ability to reproduce the well-established behavioral traits of cementitious materials and to calibrate the experimental evidence regarding the role of reinforcement at the nanoscale. The statistical sensitivity of the model, the stability of the computational algorithm, and the resulting stress-strain curves that represent the material behavior in a macroscopic sense are studied in comparison with the experimental trends.

\section{Dentrite Structure of the Solid Phase in Cementitious Gel}

After hydration, the amount of water that was originally mixed with cement occurs in two different states: chemically bound in the hydrates (nonevaporable) or occupying the volume of pores; water in larger pores (capillary) may flow out easily, whereas water in the smaller pores is held by surface forces in the internal surface area of the hydrate and may be only lost upon heating. Therefore, the entire volume of the porous phase represents space that was originally occupied by water molecules which were subsequently chemically bound in the solid phase of the cement hydrates. The origin of strength and stiffness is due to the cement hydrates. As the chemical reaction proceeds, the solid phase grows radially from the reacting cement grain. During this process, growths from adjacent grains meet and merge in space, developing a solid network of amorphous mass; in the context of the present study, the axis of a branch is modeled by a linear element although it is implicit that it is endowed by hydrate volume that provides it with solid mass; the organization and scale dimension of the hydrates depend very much on the water/binder ratio, $w_{o}$, used during the mixing. The branch-like element, which resembles the structure of a tree, is referred to in the present study as a "dendrite." Its form has been confirmed by electron-scanning microscopy (ESM) pictures of the microstructure, which show that the branches of solid mass span over gaping voids exactly like a network of truss elements. In the model, the linear elements that represent the dendrites are randomly generated from an initial, random distribution of cement grains, mimicking the chaotic distribution in nature as it is evident in ESM pictures (Figure 1). Dendrites fail in a brittle manner in axial tension whereas they are susceptible to buckling under compression due to their aspect ratio. In such a context, fibers of a scale comparable to the dendrite branches, such as carbon nanotubes (CNTs), added as mass reinforcement and properly anchored in the solid mass, add to the total number of elements bridging voids, thereby contributing (when stretched in tension) to the active links of the space truss that forms the solid phase of the cement hydrate; this may occur for fibers acting parallel to axial tension, or transversely to compression in response to Poisson-type lateral expansion of the macroscopic material [5]. Similarly, 


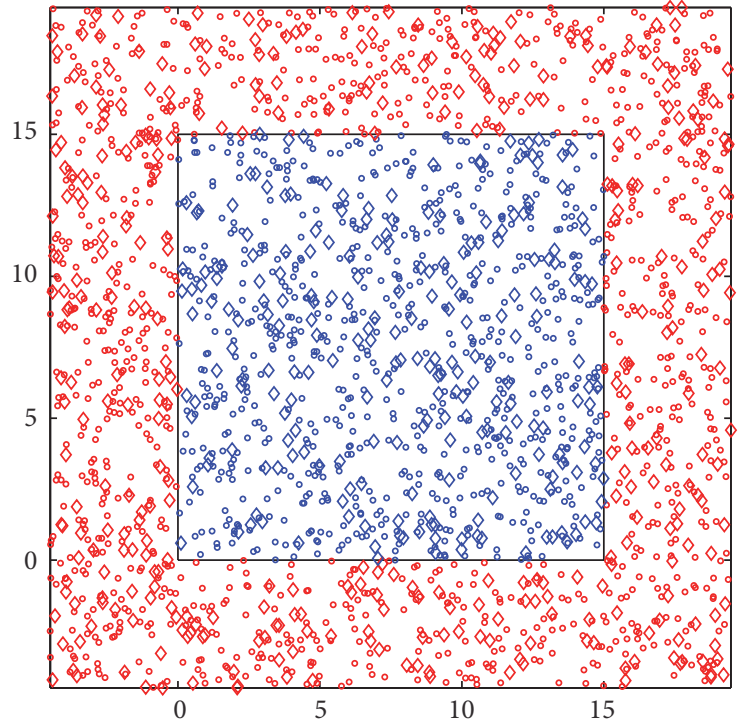

(a)

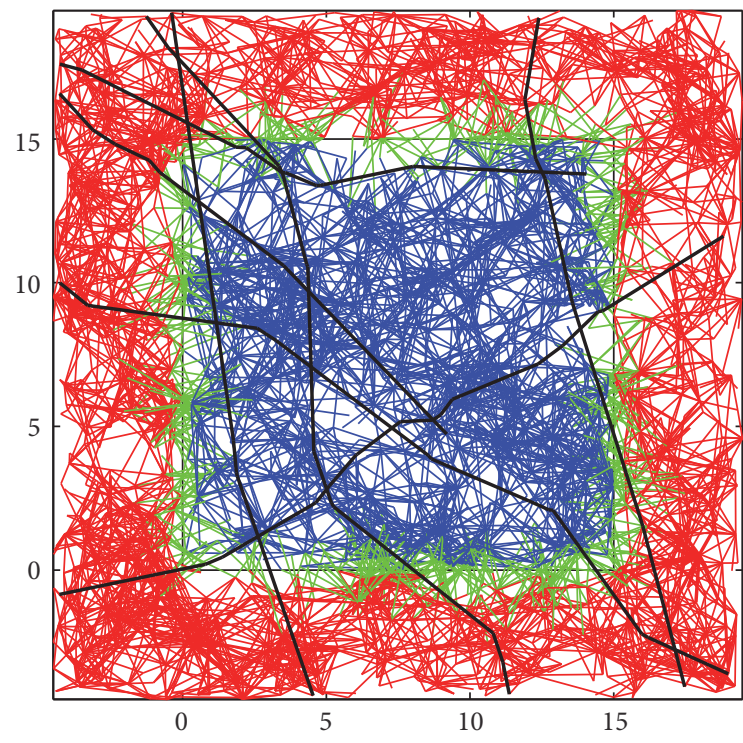

(b)

Figure 1: Discrete square space $24 \times 24 \mathrm{MHD}$. Central study region $15 \times 15 \mathrm{MHD}$ is referred to as the "box." Nodes and dendrites lie inside the box (blue) or outside the box (red) or across the boundary (green). (a) Source and target nodes for mesh generation. (b) Definition of dendrites and addition of CNTs (shown in black lines).

ESM photographs $[4,8]$ of the microstructure of CNT-reinforced materials illustrate this function of fibers: longer carbon nanotubes may even pass through several dendrites while at the same time interconnecting the solid flakes.

\section{Mean Hydraulic Distance as a Measure of Scale in the Nanostructure}

A characteristic measure of internal geometry in cementitious solids is the so-called mean hydraulic distance which is an idealized estimate of the mean separation distance between solid particles in the undisturbed (unstressed) condition. Another way to measure this distance is the diameter of the mean pore, $\delta_{o}$, since in reality a pore represents the distance between particles in the solid phase. Of course, such a measure may be misleading if seen as a deterministic geometric variable; it should rather be interpreted in light of the amorphous nature of the solid as a statistical mean distance. The mean pore size, considering that a capillary pore is idealized as a cylindrical slit with cross-sectional radius of $r_{o}$ (i.e., $\delta_{o} / 2$ ), may be estimated by the total pore volume divided by the pores' internal surface area, both being measureable and characteristic properties of the hydrate. Results from previous research [9] approximate $r_{o}$ as the ratio of the evaporable water content of the cement hydrate, $w_{e}$, divided by the internal surface area of the pore structure, $\Sigma_{\mathrm{BET}}$. Both variables are intrinsic properties of the hydrate which are expressed in terms of the water : cement ratio of the mix, $w_{o}$. The term $w_{e}$ is quantified as the pore water lost on heating to $110^{\circ} \mathrm{C}$ and is approximated as $w_{e}=14.673 w_{o} V_{m} \times$ $10^{-6}$ (in $\mathrm{m}$ ), whereas $\Sigma_{\mathrm{BET}}$ is the internal surface area of the pores, estimated as $\Sigma_{\mathrm{BET}}=3570 V_{m} \mathrm{~m}^{2} / \mathrm{g}$ of anhydrous cement. $V_{m}$ is the monolayer capacity of water of the hydrated gel, assuming that the mean diameter of a water molecule is $10.6 \mathrm{~A}^{\circ} ; V_{m}$ is estimated from the chemically bound water $w_{n}$ [9]. Thus, the hydraulic mean distance is estimated as

$$
\begin{aligned}
\delta_{o} & =2 \frac{w_{e}}{\sum_{\mathrm{BET}}}=8.22 \times 10^{-9} w_{o} \text { (in m) } \\
& \left.=8.22 w_{o} \text { (in } \mathrm{nm}\right) .
\end{aligned}
$$

For example, given a water-cement ratio, $w_{o}=0.4$, it follows that the mean pore diameter in the gel is $\delta_{o}=3.288 \mathrm{~nm}$, whereas for $w_{o}=0.6$ the characteristic distance is $\delta_{o}=4.932 \mathrm{~nm}$. Thus, based on the definition of the hydraulic mean distance as a measure of the equilibrium separation distance, a direct link between the density of packing in the cementitious matrix and mechanical strength is possible; a higher waterto-cement ratio leads to a less densely packed structure due to the increase in porosity. Clearly, the smallest unit that might be considered as a representative material sample must be in the order of few tens of nanometers so as to maintain the characteristics of the solid including its porosity while also allowing for statistical variability in the geometric arrangement of the solid phase.

\section{Discrete Model of Cement Hydrate}

4.1. Mesh Production and Generation of Dendrites. The problem is idealized in a two-dimensional plane; that is, planestress conditions are considered in the model. In principle, the model may be extended to the three dimensions, albeit at significant computational expense. The essential attributes of the solid structure of cementitious gel are emulated by abstracting the solid phase so as to represent it as linear segments spanning along the axes of the dendrites which 
emanate from a mesh of nodes which correspond to the initial position of the cement grains.

Therefore, the first step in generating the computational mesh is the random placement of cement grains in a rectangular solid section that extends in $x$ and $y$ several times the mean hydraulic distance $(\mathrm{MHD}=$ mean pore diameter $=$ $\delta_{o}$ in (1)). Each cement grain is a source node, with uniform probability of density $\mathrm{MHD}^{-2}$ (Figure 1(a)). The number of cement grains that fit in the $x-y$ space is a function of the cement content per unit volume of the mix. The generic step of the mesh-formation algorithm is to "grow" the solid structure from each partially hydrated cement grain. Next, an independent random set of uniformly distributed destination points is generated and superimposed in the test domain (Figure 1(a)). These nodes are referred to as target nodes, and they occur 3 times as frequently as the source nodes. The two sets of nodes are connected by branches that are generated randomly. However, not all possible connections are enforced. Branches represent hydration products in the form of dendrites growing outwards from the cement grains. Each pair of a source and a target node represents a potential branch, which is actually added to the model only if $\exp (-$ $\mathrm{L} / \mathrm{MHD})>\operatorname{rand}($ ), where $\mathrm{L}$ is the length of the potential branch in question and rand() is a computer-generated pseudorandom number (uniformly distributed in $[0,1]$ ). Thus, branch length is an exponentially distributed random variable with expectation MHD. However, a maximum allowed branch length is also enforced and arbitrarily set equal to $3 \mathrm{MHD}$; higher upper limits to the lengths of the dendrites have also been considered and their effect on the resulting constitutive behavior of the material has been explored. The dendrite branches are assumed to have circular cross section, the area of which is the same at all branch tips. The tip cross section is set arbitrarily equal to $10^{-8}$ in consistent units and increases linearly with distance from the tip, up to $1.2 \times 10^{-8}$ at $1 \mathrm{MHD}$ from the tip and to a maximum of $1.6 \times 10^{-8}$ at $3 \mathrm{MHD}$ from the tip, which is the maximum allowed branch length.

The mesh considered for response analysis under various boundary conditions extends over a square zone with a size that ranges between $6 \times 6$ and $30 \times 30$, in units of MHD, with $15 \times 15$ being considered a regular specimen size (this is the most common size of samples without fibers that were used to study the behavior of the cementitious material to a variety of stress states). The square zone is referred to hereon as a "box." Node and branch generation, however, occurs over a much wider area and the exterior boundary of the "box" is centered in the area of source and target node generation, as depicted in Figure 1(b). Dendrite branches that cross the boundary of the "box" are trimmed at the point of intersection with the perimeter (branches in green in Figures 1(b) and 2(b)). New nodes on the boundary of the box generated in the process (green in Figure 2(a)) are related to master nodes. This is a computational device that enables enforcement of constant principal strains (equivalent to affine kinematics) on the whole boundary of the box, while making sure that the computational area is free of boundary artifacts and as representative of "average conditions" as possible.
The dendrite material is considered linear elastic in tension and compression. Basic properties refer to the tensile stress and strain values at rupture, $\sigma_{b f}$ and $\varepsilon_{b f}$. Compressive strength at crushing is taken ten times as high; thus, compression crushing of the branch material is unlikely. Instead, the most prevalent type of failure in compression is linear elastic Euler buckling. This is followed by a brittle response cutoff. The modulus $E_{b f}$ of the dendrite material is obtained from the ratio $\sigma_{b f} / \varepsilon_{b f}$. This value is used in the subsequent study in order to normalize the value of the secant modulus of the "box" to the applied strain. (Input values used in the present study were $E_{b f}=10^{+9}, \varepsilon_{b f}=10^{-5}$, and $\sigma_{b f}=$ $10^{+4}$, in consistent units.) Finally, the branches are treated as if they were cylindrical of "average" cross section, both in force/stiffness state determination and in estimating Euler buckling loads (i.e., the paraboloid taper in the lengthwise direction of the typical dendrite is replaced for these calculations with an equivalent uniform cross section).

A similar generation procedure was used in order to define CNTs in the mesh, so as to enable a random definition of their layout in the sample specimen. Considering the usual CNTs that are currently available on the market, their length is in general about two orders of magnitude longer than dendrites and one order of magnitude longer than the sample region. So, CNTs are defined in the greater generation area and then only those that cross the boundary of the "box" are considered in studying the mechanics of the state of stress of the composite (Figures 1(b) and 2(b)). In placing and orienting the CNTs and in light of the ESM photographic material that shows them to be generally curved in their final position $[4,5]$, they are modeled as multilinear segments in the material space. This is consistent with the fact that CNTs may be curved in the fresh mix state and "frozen" in that position after hardening. To achieve this from a computational perspective, in the present model, the fibers were assumed to interact with the network of hydrates by being attached to target nodes. Therefore, a typical CNT may comprise a multisegment element attached to several nodes. This model recognizes that the macroscopic effects imparted by the CNTs will be primarily controlled by the volumetric ratio of nanofibers in the mix. The discrete number of fibers within the extended specimen, before trimming, is an integer approximation of their cross-sectional area times their expected length divided by the volume of the extended specimen. The extreme variability of fiber density is expected. This is more so for higher fiber cross-sectional areas, since very few fibers are generated (in some cases as few as five) and even fewer lie within the specimens eventually analyzed. The actual strength and stiffness parameters of the fibers are not particularly relevant in compression as fibers are considered inactive in that direction. Fibers function as onesided springs that are only engaged in tension. Their modulus of elasticity is at least one order of magnitude higher than that of the hydrate, taken here as $E_{\mathrm{fib}}=1 e+10$ in consistent units.

4.2. Performance of the Idealized "Box" Area under Load. In the present section, the mechanical behavior of the "box" model is studied under various controlled boundary 


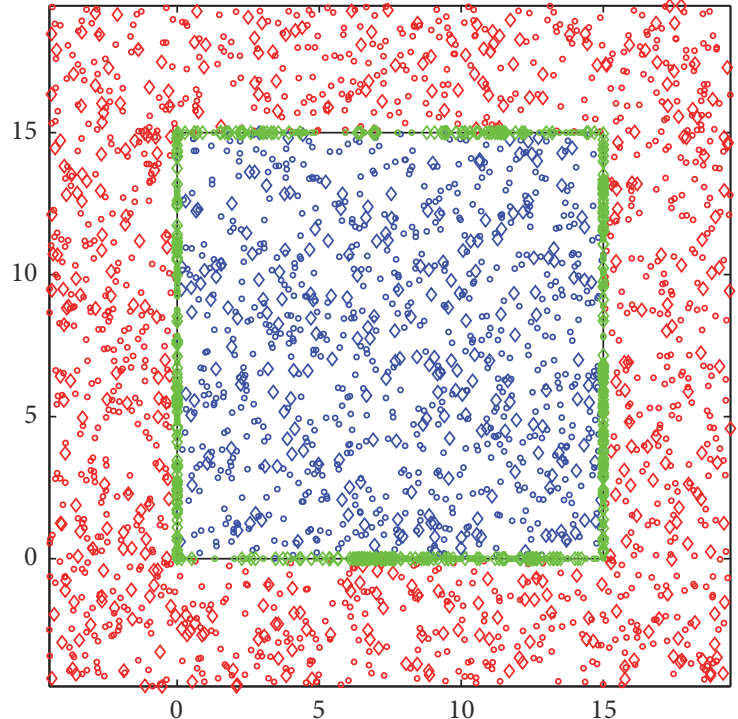

(a)

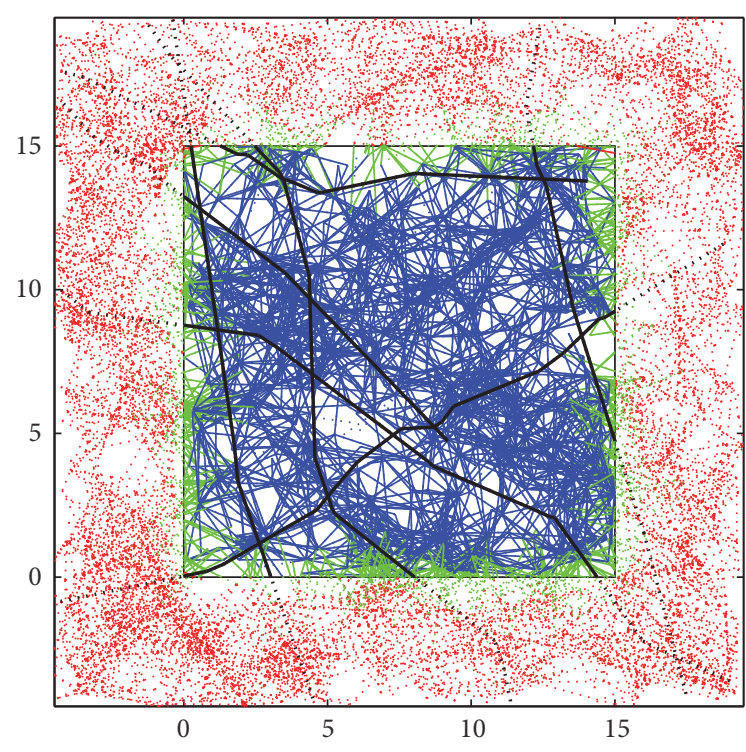

(b)

Figure 2: Trimming of the $15 \times 15$ MHD "box." (a) New boundary nodes (green) are created where branches cross the perimeter of the box. (b) Trimming of elements to the "box." Dashed lines represent trimmed dendrites (red) and CNTs (black). Space not occupied by the solid structure represents the voids (pores) of the material.

conditions, with the objective of identifying and interpreting the contribution of the CNTs in the apparent properties of the reinforced cementitious matrix. Analysis in $2 \mathrm{D}$ is conducted using constant strain increments, $\Delta \varepsilon_{2}=\varepsilon_{b f} / 100$ or $\Delta \varepsilon_{2}=\varepsilon_{b f} / 200$, along the direction of displacement control (i.e., along geometric axis 2 in Figure 1), with tolerance of $10^{-6}$ in both displacement values and loads. A direct stiffness assembly process is used to define the current form of the equation of static equilibrium. Solution is performed using standard nonlinear system solvers (Newton type). With refinement of increments, convergence is improved; the last converged configuration is used as the starting point in each new increment. Note that divergence of the algorithm has occurred in certain cases, due to local singularities that arise after failure of some dendrites.

Loading on the model may be conducted under either load or displacement control using the master nodes which control the kinematics of the boundary nodes as described in Section 4.1. An important reference point that may be used to assess the validity of the model is the relevance of calculated response of the reinforced composite under uniaxial compression and tension to the observed experimental trends $[3,5]$. Analysis of the uniaxial stress state is conducted under displacement control along axis 2 that corresponds to the axis of load application. In the transverse direction, the "box" is left to expand or contract freely with the condition that the corresponding stress resultants are zero. Similarly, analysis of the uniaxial strain state (tension or compression along axis 2) is conducted by requiring that zero translation occurs in the transverse direction (boundary conditions along axis 1). Finally, pure shear strains are enforced by requiring the translation in the transverse direction (axis 1) to be the opposite of the controlled displacement (along axis
2). CNT-reinforced samples are only tested under uniaxial stress or strain conditions; further experiments are underway to calibrate the CNT-model performance under arbitrary states of stress. Figures 4(a) and 4(b) plot the corresponding stress-strain relationships that characterize the response of the composite (as does Figure 3 for plain paste). Here, each principal strain is obtained by dividing the displacement with the corresponding "box" dimension, and each stress is defined as the ratio of the resultant traction force divided by the area presented to load (=breadth of the "box" times its thickness of one MHD). Plots include smeared axial-stressstrain relationship in the direction of loading. In all cases, the stress terms are nondimensionalized with respect to the material properties of the branches, that is, with respect to the ideal properties of the cementitious solid. Furthermore, for the sake of comparison of results from specimens of different material densities, stresses are further normalized by division with the volume ratio of the gel in the "box."

Each time the program is run with the same input values, a different random truss is created, owing to the random generation process. Hence, two otherwise identical execution instances of the algorithm will not lead to identical results. Variability occurs not only in the net magnitude of the values but also in the state of convergence of the algorithm at various levels of applied displacement. This is reflected in the occasional digressions from the calculated stressstrain curves, most of which are "local" and swiftly return to the backbone curve. Nevertheless, all calculated stressstrain envelopes (Figures 3, 4(a), and 4(b)) have very clear backbones that follow qualitatively the expected shape of compressive and tensile stress-strain response of semibrittle materials as is known from experimental experience $[1,2$, $4,5,9,10]$. Even for loading conditions that do not give 


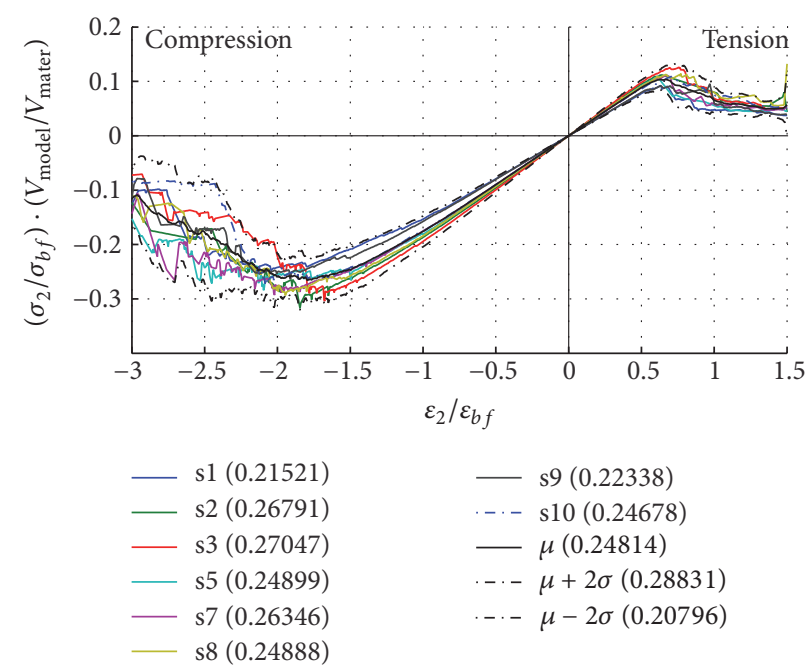

(a)
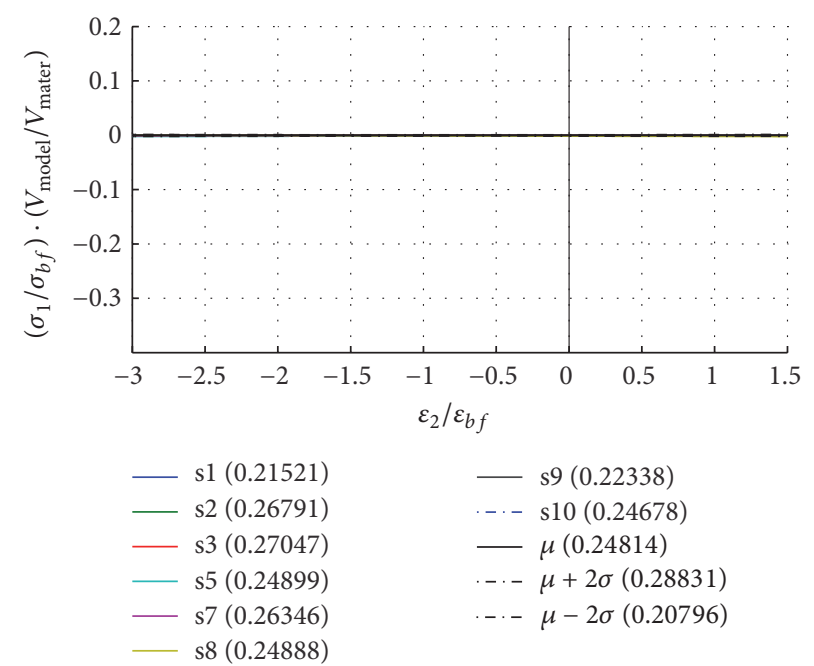

(c)

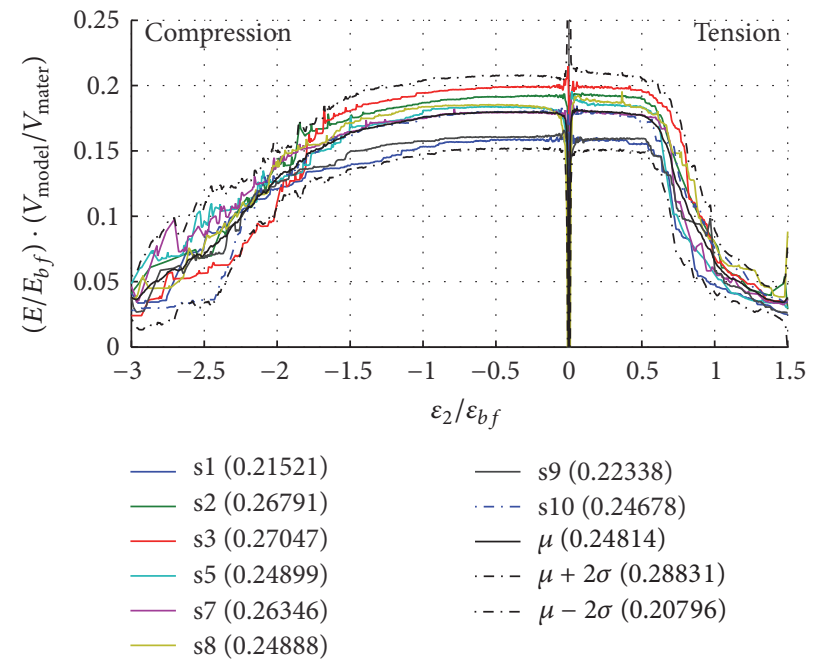

(b)
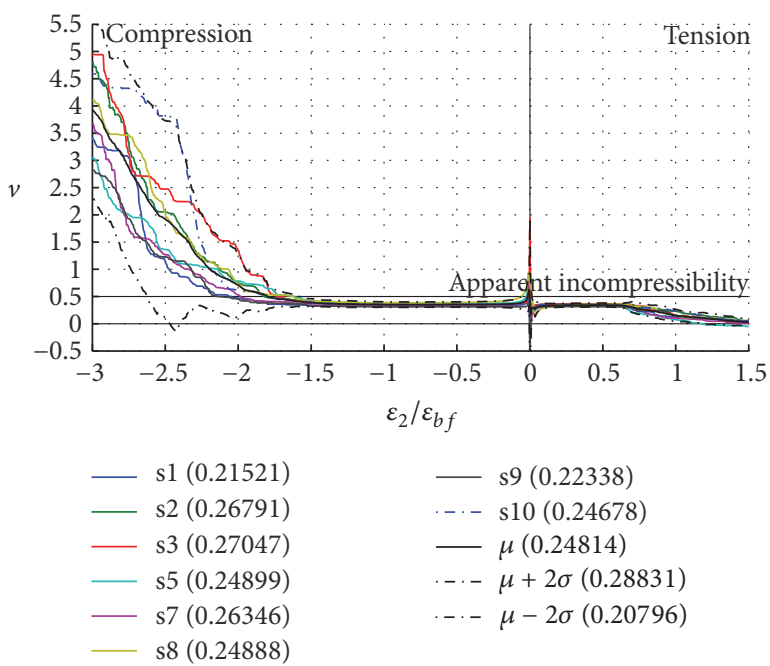

$$
\begin{aligned}
& -s 9(0.22338) \\
& --s 10(0.24678) \\
& -\mu(0.24814) \\
& -\cdot \mu+2 \sigma(0.28831) \\
& -\cdot-\mu-2 \sigma(0.20796)
\end{aligned}
$$

(d)

FIGURE 3: Individual response curves of unreinforced discrete specimens $(15 \times 15 \mathrm{MHD})$ to uniaxial plane-stress loading, along with sample mean response curves (solid black line) and $2 \sigma$-envelopes (dashed dotted black line). Stresses and moduli of elasticity are made comparable across samples by division with the observed volume density $V_{\text {mater }} / V_{\text {model }}$ of each specimen (given in parenthesis in the legend). (a) Stress in direction of loading. (b) Secant modulus of elasticity. (c) Stress across loading (zero throughout). (d) Secant Poisson ratio (apparent incompressibility observed at limit point in compression).

rise to macroscopic stretching, internal equilibrium of the random truss dictates that many elements must be in tension, and usually these fail first (leading to redistribution of tension to decreasingly capable elements). The characteristic of the softening postpeak branch occurs when tensile fracture of dendrites orients in the direction of prevalent tension, followed by an avalanche of buckled dendrites oriented in the direction of prevalent compression.

4.3. Discussion of Results. The parametric performance of the discrete material model is illustrated in the graphs of Figures 3, 4(a), and 4(b). Each figure comprises four graphs that present in $2 \mathrm{D}$ the relationship between smeared stresses and the axial and lateral strain for the case study considered.
The $x$-axis represents the applied controlling strain (i.e., the displacement normalized with the "box" dimensions so as to have the physical significance of infinitesimal strain), $\varepsilon_{2}$; this variable is normalized further with the rupture strain of the basic dendrite material, $\varepsilon_{b f}$. In the four graphs shown, the $y$-axis has the following significance: in case (a), it is the average value of stress, $\sigma_{2}$, which is collinear to the applied strain $\varepsilon_{2}$; again, $\sigma_{2}$ is plotted after being normalized by $\sigma_{b f}$ and by the volume fraction of gel solid in the "box." In case (c), the plotted variable is the lateral stress $\sigma_{1}$, after having been normalized in the same manner. In case (b), the $y$-axis represents the secant modulus of elasticity normalized by the dendrite material modulus, $E_{b f}$, and by the volume fraction of gel solid in the "box." In case (d), the plotted variable is the secant value of Poisson's ratio, $v$. 


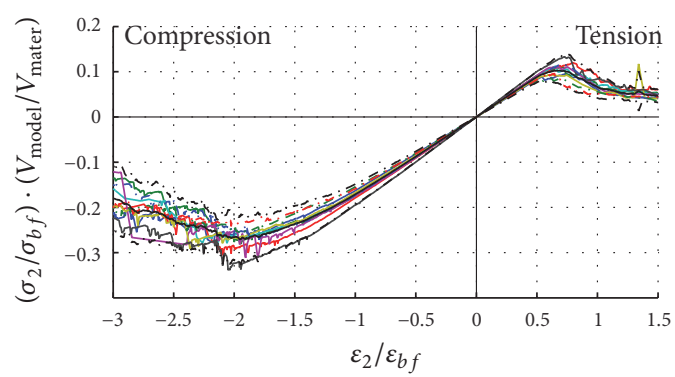

(A)

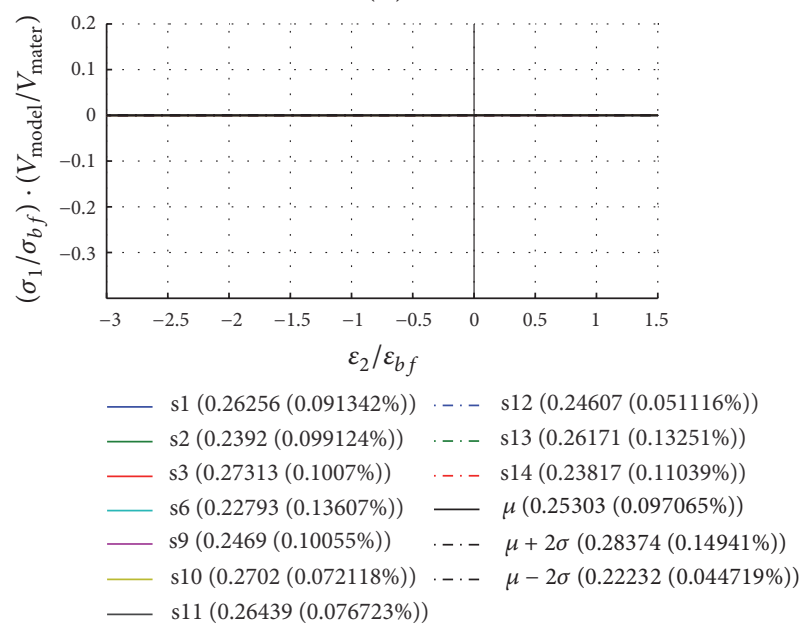

(C)

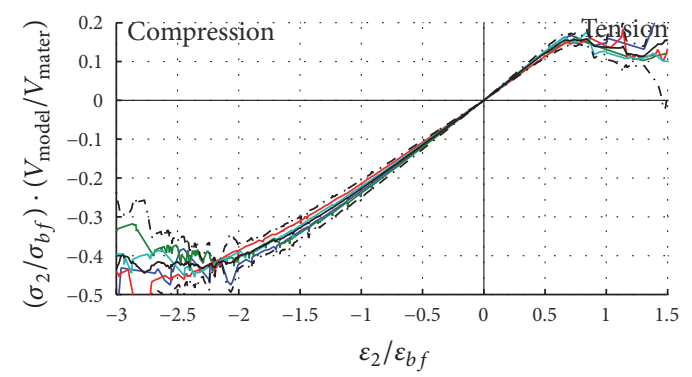

(A)

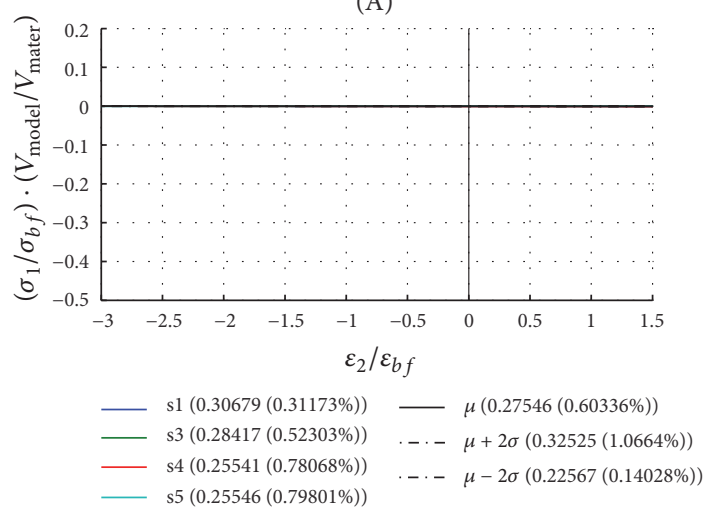

(C)

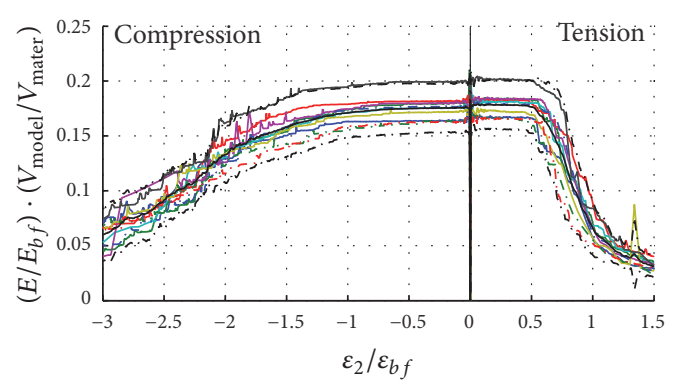

(B)
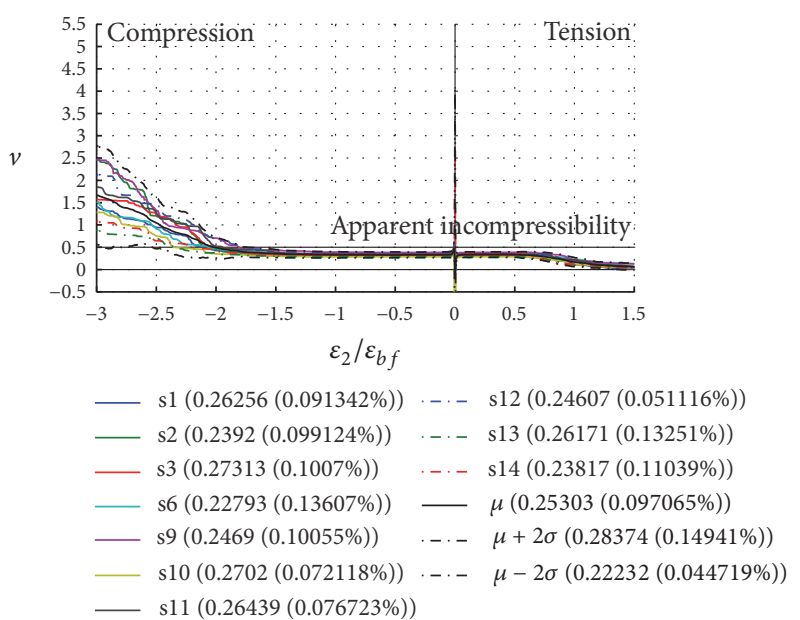

(D)

(a)

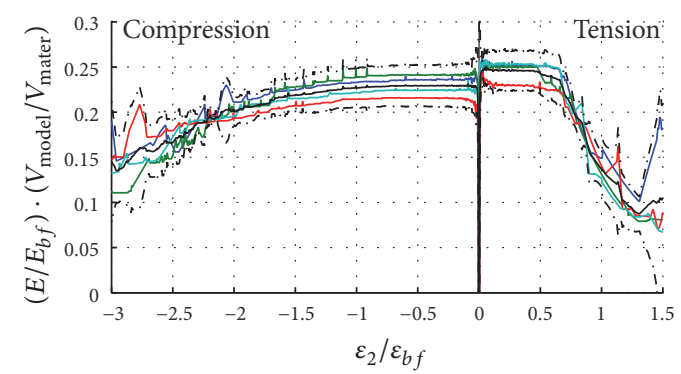

(B)
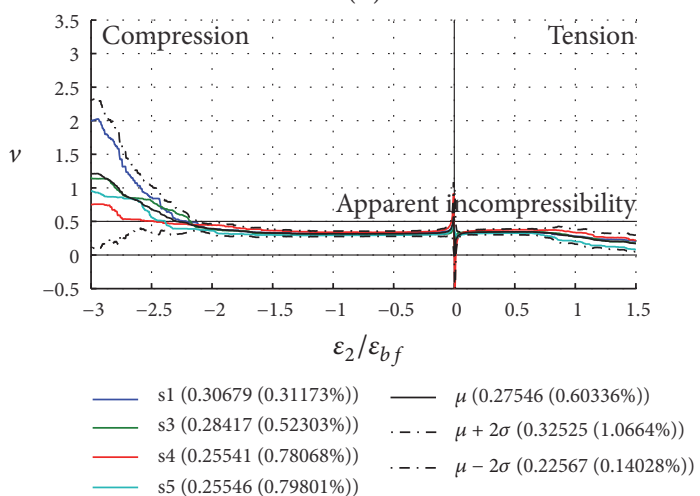

(D)

(b)

FIGURE 4: Individual response curves, along with sample mean response curves (in solid black) and 2 $\sigma$-envelopes (in dashed dotted black), for uniaxial plane-stress loading of discrete specimens $(15 \times 15 \mathrm{MHD})$ reinforced with CNTs. Stresses and moduli of elasticity are divided by the observed volume density $V_{\text {mater }} / V_{\text {model }}$ of each specimen (given in parenthesis in the legend, along with the actual CNT content per solid volume of specimen). (A) Stress in direction of loading. (B) Secant modulus of elasticity. (C) Stress across loading (zero throughout). (D) Secant Poisson ratio (apparent incompressibility observed at limit point in compression). The two groups differ with respect to the targeted CNT content, which is (a) $0.1 \%$ per volume of paste and (b) $1 \%$ per volume of paste. 
Each example material and stress state is studied with several analytical specimens, as discussed earlier. Therefore, the bundle of curves depicted in any given type of plot has been obtained by randomly generating a study "box" each time and repeating the solution using identical input values for each run. So, samples 1, 2, 3, and so forth represent cases that are in principle identical but are practically different, since they have been randomly generated. Note that the observed variability between curves is expected and represents the analytical analogue of the statistical variability witnessed in real lab work, whenever a group of identical specimens is physically tested. Each specimen is codified in the legend of the figure by a composite ID, which includes the specimen number, its volume density, and the corresponding CNT volume ratio $\left(V_{\mathrm{CNT}} / V_{\text {mater }}\right)$, if any; here $V_{\mathrm{CNT}}$ and $V_{\text {mater }}$ are the volume of CNTs and the volume of hydrates, respectively, contained in a "box." Volume density is defined as the ratio of the volume of solid material in the "box," $V_{\text {mater }}$, to the volume of the "box" itself (i.e., $\left.V_{\text {model }}=A_{\text {model }} \times \mathrm{MHD}\right)$. For example, a code of s1 $(0.26256(0.091342 \%))$ refers to specimen number 1 from the batch in question, containing dendrites that account for $26.3 \%$ of its volume (which is $15 \times 15 \times 1 \mathrm{MHD}^{3}$ in all three figures). This number $(0.26256)$ divides the stress and stiffness values measured from the specimen to make them comparable with those of other specimens (of different density). The measured volume ratio of CNTs to dendrites is $0.091 \%$ (whereas the targeted value for the batch is $0.1 \%$ ). Note that if a specimen number is missing in the sequence, this implies that global divergence occurred in the simulation of its response, so its results are not available.

An important open issue of the investigation is to determine whether the computational results are susceptible to mesh sensitivity. Towards this objective, several groups of specimens of plain paste (nominally differing only in box dimensions) were examined in uniaxial compression, uniaxial tension, and pure shear. Results are summarized in Table 1. Subscripts $c t$ and $c c$ refer to tensile and compressive terms of the cementitious composites, respectively, whereas $c v$ refers to shearing of the same (applied kinematically under shear-strain control). In the first column, $N$ represents the linear dimensions of numerical specimens ranging from 6 to $30 \mathrm{MHD}$. Mean values of limit-point responses for each group of samples studied are listed (with the coefficient of variation given in parentheses). Clearly, scale effects are significant and may be attributed both to the nature of the model (brittle dendrites making up the solid structure) and to numerical considerations (kinematic boundary constraints). In all simulations, the (secant) Poisson ratio is strikingly consistent, regardless of sample parameters or loading considered. Its initial values are estimated at $v=1 / 3$ and remain approximately constant in tension tests up to the limit point. Its effective value rises to $v=0.5$ near the limit points in compression regardless of the boundary condition, consistent with experimental results from previous studies on confined plain concrete [9-11]. It is therefore concluded that using $\nu=$ 0.5 as the criterion of compressive failure complies with the experimental evidence.

It was observed that, in cases dominated by tensile strain, behavior is almost linear up to the limit point. For $15 \times 15 \mathrm{MHD}$ specimens, the coordinates of the limit point are as follows: (a) in uniaxial tension, $\bar{\varepsilon}_{c t}=0.67( \pm 11 \%)$ with $\bar{\sigma}_{c t}=0.11$ $( \pm 10 \%)$; (b) in kinematic shearing, $\bar{\varepsilon}_{c v}=0.68( \pm 10 \%)$ with $\bar{\sigma}_{c v}=0.077( \pm 10 \%)$; (c) in uniaxial stretching (where it is found that $\sigma_{1} \approx \sigma_{2} / 3$ up to the limit point) at the onset of the tensile stress-strain postpeak branch, $\bar{\varepsilon}_{c t}=0.62( \pm 8 \%)$ with $\bar{\sigma}_{c t}=0.11( \pm 5 \%)$; and (d) in biaxial tension with $\sigma_{1} \approx \sigma_{2} / 2$, $\bar{\varepsilon}_{c t}=0.63( \pm 9 \%)$ with $\bar{\sigma}_{c t}=0.12( \pm 12 \%)$. The results indicate failure at an ultimate tensile stress in loading cases with $\sigma_{1} \sigma_{2} \geq$ 0 and failure at an ultimate tensile strain in loading cases with $\sigma_{1} \sigma_{2} \leq 0$. Transition between the two failure criteria occurs at the point of uniaxial tension with $\sigma_{1} \sigma_{2}=0$, where both $\sigma_{2}=f_{c t u}$ and $\varepsilon_{2}=\varepsilon_{c t u}$ apply.

Consistent with the apparent uniaxial stress-strain response recorded during tests, analyses of computational samples where compressive stresses dominate failure are marked by pronounced nonlinearity before the limit point. For $15 \times 15 \mathrm{MHD}$ specimens and failure defined by $v \approx 0.5$, the following results are obtained for the limit point: (a) under uniaxial compression, $\bar{\varepsilon}_{c c}=-1.90( \pm 6 \%)$ with $\bar{\sigma}_{c c}=-0.30$ $( \pm 3 \%)$; (b) under uniaxial contraction, $\bar{\varepsilon}_{c c}=-4.83( \pm 20 \%)$ with $\bar{\sigma}_{c c}=-0.64( \pm 17 \%)$, where $\sigma_{1}=\sigma_{2} / 2$ at failure; and (c) under biaxial compression with $\sigma_{1}=\sigma_{2} / 2, \bar{\varepsilon}_{c c}=-5.05$ $( \pm 10 \%)$ with $\bar{\sigma}_{c c}=-0.69( \pm 21 \%)$. Note that the last two cases lead to essentially the same limit point (the differences in both stress and strain being statistically insignificant) but trace different paths in stress and strain. Note that, consistent with the experimentally documented effects of confinement, this limit point is roughly 2.3 times higher in stress and 2.8 times higher in strain than the one in uniaxial compression. Also, the ratio of compressive to tensile strength $\left(\sigma_{c c} / \sigma_{c t}\right)$ is about 3, owing to the absence of aggregates in the model (the ratio is appropriate for paste only as modeled here).

Analysis under kinematic shearing (i.e., external load is applied under shear-strain control in a state of pure shear strain) yields a limit state of stress where the compressive stress is higher than the tensile one, by $5 \%$ in one direction of loading and by $13 \%$ in the reverse. If this difference were due to the anisotropy of the random specimens, it should have roughly zero mean value over the sample. Since this is not the case, some disintegration is clearly present at the limit point. Using all samples, the estimated mean values are $\bar{\varepsilon}_{c v}=$ $0.68( \pm 10 \%)$ with $\bar{\sigma}_{c v}=-0.077( \pm 8 \%)$, where $\bar{\sigma}_{c c v}=-0.080$ $( \pm 8 \%)$ and $\bar{\sigma}_{c t v}=+0.074( \pm 8 \%)$. The physical significance of this result is that the limit value of shear strength is $70 \%$ of the tensile strength $\bar{\sigma}_{c t}=0.11( \pm 10 \%)$, which is within the bounds of the expected magnitude.

To assess the contribution of CNT reinforcement on the mechanical properties of the material, different CNT contents were considered in the study; nominal CNT volumetric ratios, calculated with reference to the final material volume, were targeted to values of $1 \%, 0.5 \%$, and $0.1 \%$. These are usual contents that have been tried in tests [5-7] (parameter $A_{\text {fib }}$ represents the cross-sectional area of the CNTs). Due to the manner of their generation (see Section 3), the actual yield of CNTs in the mix differed from the targeted ratios (Table 2).

Comparison of results at these levels of reinforcement with results from plain paste samples indicates the following effects (Figures 4(a) and 4(b)). (a) When using unrealistically 
TABLE 1: Summary of mesh sensitivity study: normalized stresses and strains at limit points.

\begin{tabular}{lcccccc}
\hline Mesh size in MHD multiples $N$ & $\bar{\varepsilon}_{c t}=\frac{\varepsilon_{c t}}{\varepsilon_{b f}}$ & $\bar{\sigma}_{c t}=\frac{\sigma_{c t}}{\sigma_{b f}} \frac{V_{\text {model }}}{V_{\text {mater }}}$ & $\bar{\varepsilon}_{c c}=\frac{\varepsilon_{c c}}{\varepsilon_{b f}}$ & $\bar{\sigma}_{c c}=\frac{\sigma_{c c}}{\sigma_{b f}} \frac{V_{\text {model }}}{V_{\text {mater }}}$ & $\bar{\varepsilon}_{c v}=\frac{\varepsilon_{c v}}{\varepsilon_{b f}}$ & $\bar{\sigma}_{c v}=\frac{\sigma_{c v}}{\sigma_{b f}} \frac{V_{\text {model }}}{V_{\text {mater }}}$ \\
\hline 6 & $0.80( \pm 12 \%)$ & $0.15( \pm 13 \%)$ & $-2.30( \pm 8 \%)$ & $-0.38( \pm 15 \%)$ & $-2.12( \pm 13 \%)$ & $-0.35( \pm 16 \%)$ \\
9 & $0.75( \pm 8 \%)$ & $0.13( \pm 11 \%)$ & $-2.09( \pm 6 \%)$ & $-0.34( \pm 10 \%)$ & $-1.99( \pm 10 \%)$ & $-0.31( \pm 10 \%)$ \\
15 & $0.68( \pm 11 \%)$ & $0.11( \pm 10 \%)$ & $-1.92( \pm 5 \%)$ & $-0.30( \pm 3 \%)$ & $-1.90( \pm 6 \%)$ & $-0.30( \pm 3 \%)$ \\
21 & $0.58( \pm 6 \%)$ & $0.091( \pm 5 \%)$ & $-1.89( \pm 3 \%)$ & $-0.25( \pm 8 \%)$ & $-1.84( \pm 5 \%)$ & $-0.25( \pm 8 \%)$ \\
30 & $0.57( \pm 6 \%)$ & $0.088( \pm 5 \%)$ & $-1.74( \pm 4 \%)$ & $-0.23( \pm 3 \%)$ & $-1.72( \pm 4 \%)$ & $-0.23( \pm 4 \%)$ \\
\hline
\end{tabular}

TABLE 2: Calculated effect of CNT reinforcement in the cementitious matrix.

\begin{tabular}{lcccc}
\hline Target CNT per vol. & Actual CNT per vol. & $\sigma_{c t}$ increase & $\sigma_{c c}$ increase & $E$ increase \\
\hline $1 \%$ & $0.3 \%-0.8 \%$ & $25 \%$ & $40 \%$ & $30 \%$ \\
$0.5 \%$ & $0.28 \%-0.41 \%$ & $10 \%$ & $10 \%$ & $10 \%$ \\
$0.1 \%$ & $0.03-0.1 \%$ & - & - & $1 \%$ \\
\hline
\end{tabular}

high CNT content (a theoretical limit), the peak load-bearing capacity is effectively doubled, presenting the behavior of passively confined concrete (Figure 4(b)); note that past work has illustrated the role of CNT or CNF reinforcement as effective internal confinement $[5,11]$. This is quantified not only by the increased compressive strength of the composite, but also by the reduced dilation rate (apparent values of the secant Poisson ratio, $\nu$ ). It is worth noting that the sample variability in this respect is very low (less than 10\%) confirming that the mechanistic confining contribution of the fibers is a consistent, repeatable finding. For lower ratios, the effects of the CNT addition on stiffness and strength of the composite are proportionately attenuated becoming insignificant for very low amounts of CNT content, although the modulus of elasticity in tension is higher than that in compression, underscoring the CNT contribution even if the CNT contents are low (Figure 4(a)). In all cases, however, the postlimit behavior was significantly improved, marked again by smaller values of apparent secant $v$ as compared to the unreinforced counterpart. This behavior is the same as that of FRC under unconfined compression [1] and is very similar to that of confined conventional concrete $[9,11]$; a mechanisticbased interpretation of the effective confinement provided by CNT fibers in the gel has been derived and corroborated with experimental results in [5]. Generally, better overall convergence and stability of deformation were observed. The CNT-reinforced samples were able to maintain 35\% to $40 \%$ of their peak load-bearing capacity even at very high levels of sample deformation, again illustrating the confining effect imparted by the CNT reinforcement in the cementitious matrix. At that stage, the CNTs function as continuity reinforcement where load transfer may follow a roundabout path through the fibers if a cluster of dendrites has encountered either rupture or buckling.

\section{Conclusions}

This paper presents results from computational simulations of the mechanical behavior of cementitious matrices reinforced by the addition of carbon nanotubes. These additives operate at the nanoscale of the matter and are intended to control the creation of flaws (cracks) within the gel structure, well before these may propagate to higher geometric scales (micro- and mesoscales), where larger, visible steel or synthetic fibers may operate. Simulations are carried out using a novel discrete representation of the nanostructure of the gel, which has been developed in the present paper from first principles. In deriving the model, the intent was to reproduce and highlight the interactions occurring between cementitious material particles and nanoreinforcement such as CNTs and CNFs. In this regard, a novel approach is used to model the hydrates as randomly generated dendrites emanating from partially hydrated cement grains and intertwining to create load and resistance paths randomly oriented in the solid. Dendrite geometric properties and cement grain positioning are randomly generated, so that responses obtained are quantified by the mean values and dispersion as occurs in laboratory testing of several identical specimens. The model provides a testbed for the study of the material behavior under stress and strain. For this purpose, the material model is subjected to constraints that mimic various controlled laboratory experiments that are conducted for material characterization (uniaxial tension/compression, uniaxial stretching/contraction, and pure kinematic shear). Calibrated to fit the macroscopic physical characteristics of the matrix (density and mean pore size), the model reproduces successfully several known macroscopic behavioral traits of cementitious materials, including the characteristics of the yield and failure surfaces under states of plane stress/strain. The discrete model is combined with a random network of CNTs of known geometric and mechanical properties to computationally reproduce the parametric sensitivities and measured properties of nanoreinforced cement-based composites successfully. It is shown that CNTs effectively confine the material passively, leading to increased strength and stiffness in compression with a much slower rate of degradation and lateral dilation (phenomenological Poisson's ratio), consistently with experimental evidence of 
a significant confining effect occurring in the microstructure of the matrix and effectively increasing the apparent material stiffness $[1,4,5,11]$.

\section{Conflicts of Interest}

The authors declare that there are no conflicts of interest regarding the publication of this article.

\section{Acknowledgments}

This research was cofinanced by the EU (European Social Fund, ESF) and Greek national funds through the Operational Program "Education and Lifelong Learning" of the National Strategic Reference Framework (NSRF), Research Funding Program: “Thales-Democritus University of ThraceCenter for Multifunctional Nanocomposite Construction Materials" (MIS 379496).

\section{References}

[1] S. J. Pantazopoulou and M. Zanganeh, "Triaxial tests of fiberreinforced concrete," Journal of Materials in Civil Engineering, vol. 13, no. 5, pp. 340-348, 2001.

[2] G. Y. Li, P. M. Wang, and X. Zhao, "Mechanical behavior and microstructure of cement composites incorporating surfacetreated MWCNTs," Carbon, vol. 43, pp. 1239-1245, 2005.

[3] M. S. Konsta-Gdoutos, "Nanomaterials in self-consolidating concrete: a state-of-the-art review," Journal of Sustainable Cement-Based Materials, vol. 3, no. 3-4, pp. 167-180, 2014.

[4] Z. S. Metaxa, M. S. Konsta-Gdoutos, and S. P. Shah, "Carbon nanotubes reinforced concrete," ACI Special Publication, vol. 276, no. 2, pp. 11-19, 2009.

[5] N. D. Archontas and S. J. Pantazopoulou, "Microstructural behavior and mechanics of nano-modified cementitious materials," Advances in concrete construction, vol. 3, no. 1, pp. 15-37, 2015.

[6] P. A. Danoglidis, M. S. Konsta-Gdoutos, E. E. Gdoutos, and S. P. Shah, "Strength, energy absorption capability and self-sensing properties of multifunctional carbon nanotube reinforced mortars," Construction and Building Materials, vol. 120, pp. 265-274, 2016.

[7] E. E. Gdoutos, M. S. Konsta-Gdoutos, P. A. Danoglidis, and S. P. Shah, "Advanced cement based nanocomposites reinforced with MWCNTs and CNFs," Frontiers of Structural and Civil Engineering, vol. 10, no. 2, pp. 142-149, 2016.

[8] L. I. Nasibulina, I. V. Anoshkin, S. D. Shandakov et al., "Direct synthesis of carbon nanofibers on cement particles," Transportation Research Record, vol. 2142, pp. 96-101, 2010.

[9] S. J. Pantazopoulou and R. H. Mills, "Microstructural aspects of the mechanical response of plain concrete," ACI Materials Journal, vol. 92, no. 6, pp. 605-616, 1995.

[10] I. Imran and S. J. Pantazopoulou, "Experimental study of plain concrete under triaxial stress," ACI Materials Journal, vol. 93, no. 6, pp. 589-601, 1996.

[11] S. P. Tastani, M. S. Konsta-Gdoutos, S. J. Pantazopoulou, and V. Balopoulos, "The effect of carbon nanotubes and polypropylene fibers on bond of reinforcing bars in strain resilient cementitious composites," Frontiers of Structural and Civil Engineering, vol. 10, no. 2, pp. 214-223, 2016. 


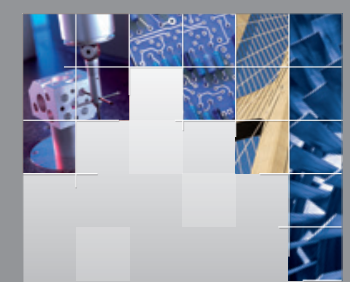

\section{Enfincering}
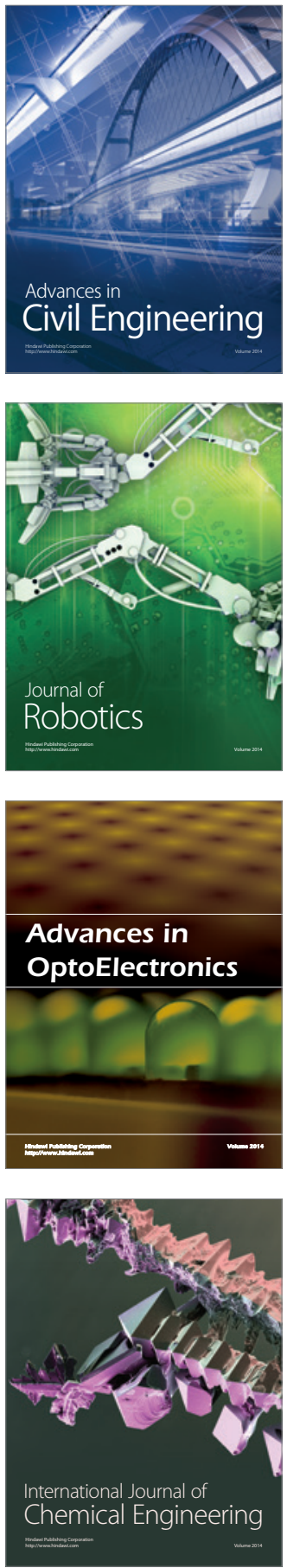

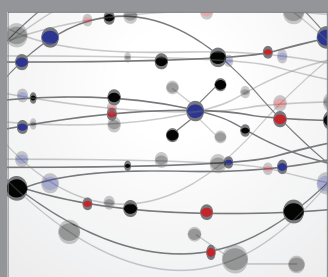

The Scientific World Journal

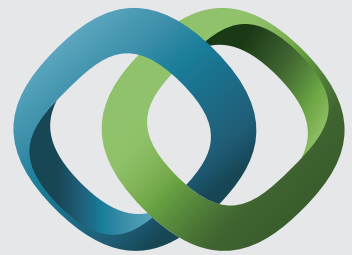

\section{Hindawi}

Submit your manuscripts at

https://www.hindawi.com
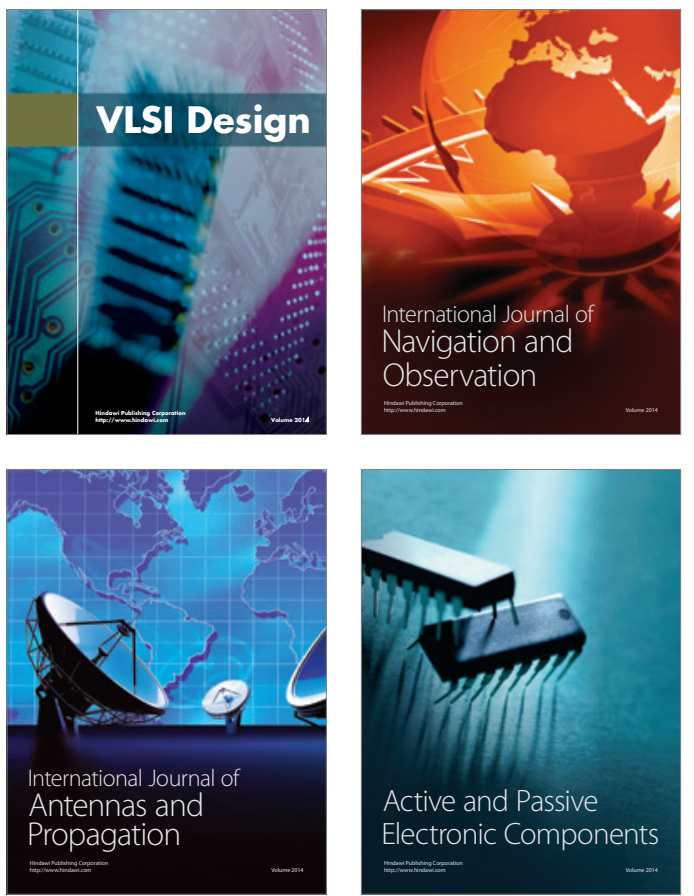
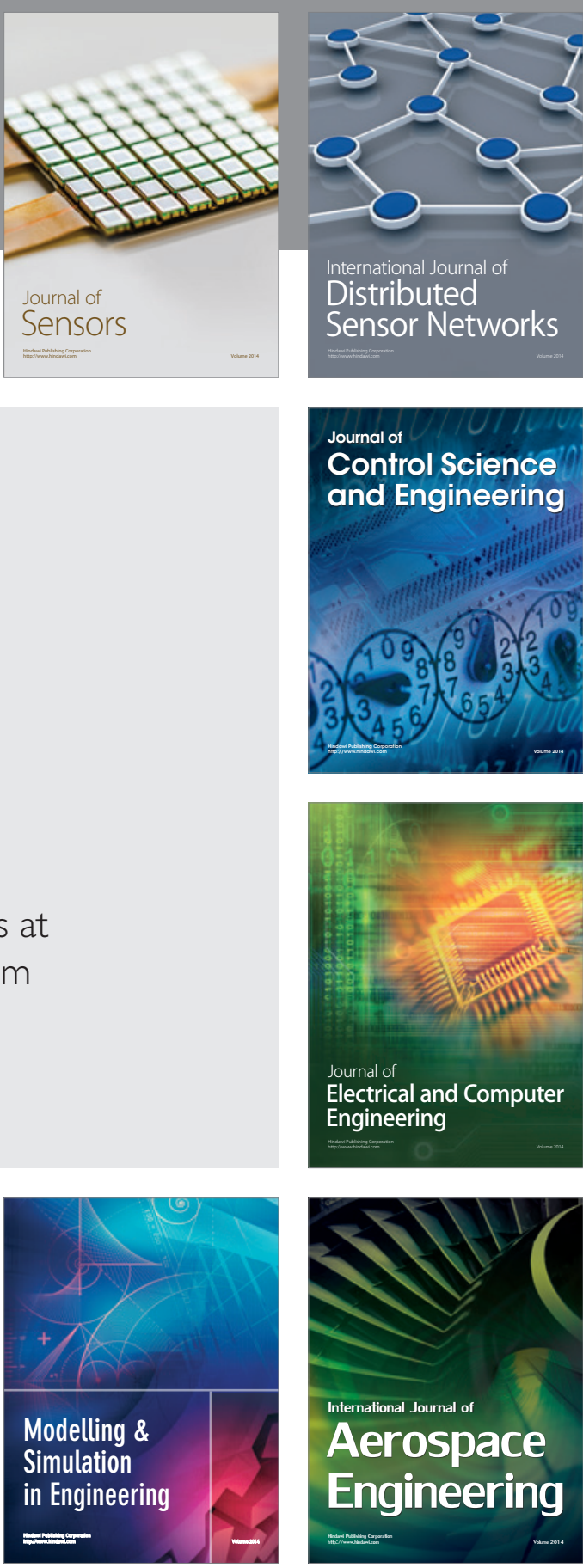

International Journal of

Distributed

Sensor Networks

$-$

Joumal of

Control Science

and Engineering
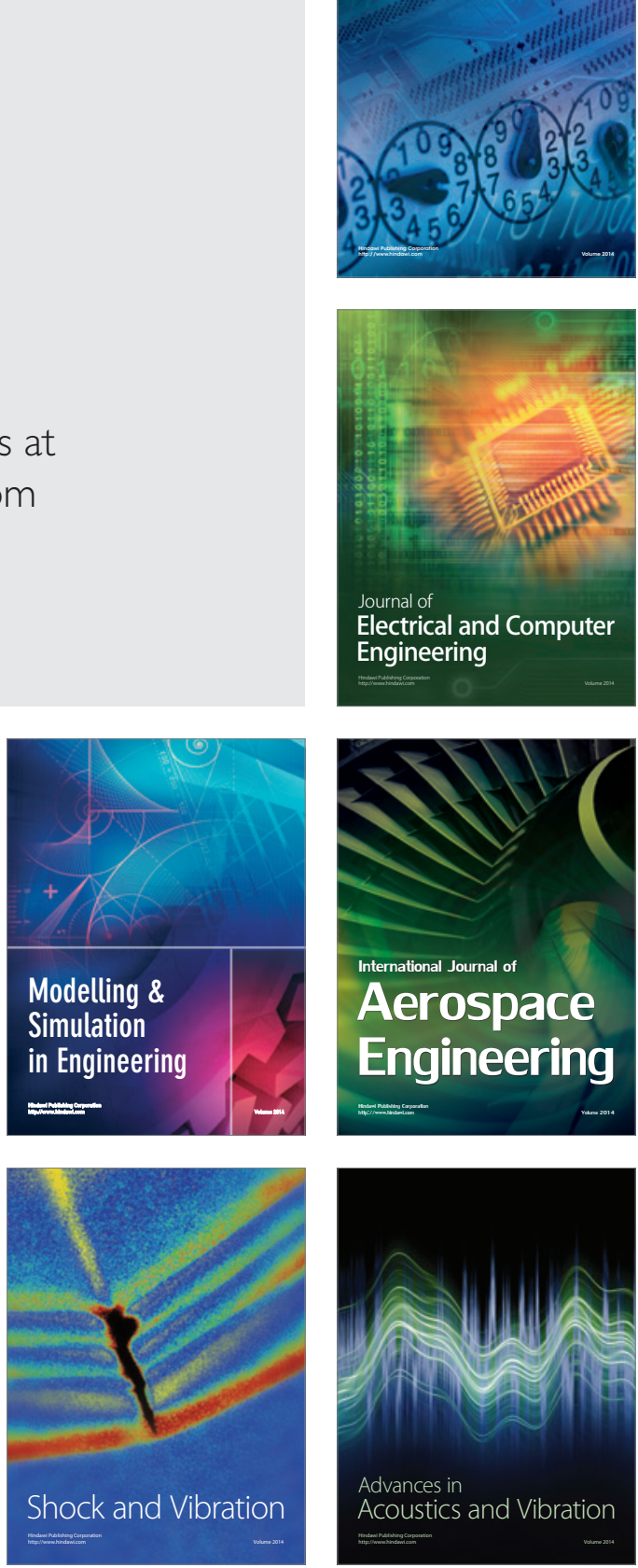